\title{
Epirubicin Hydrochloride
}

National Cancer Institute

\section{Source}

National Cancer Institute. Epirubicin Hydrochloride. NCI Thesaurus. Code C474.

The hydrochloride salt of the 4'-epi-isomer of the anthracycline antineoplastic antibiotic doxorubicin. Epirubicin intercalates into DNA and inhibits topoisomerase II, thereby inhibiting DNA replication and ultimately, interfering with RNA and protein synthesis. This agent also produces toxic free-radical intermediates and interacts with cell membrane lipids causing lipid peroxidation. 